\title{
Editorial
}

\section{The novel influenza a ( H1N1) infection in infants and children}

\author{
Musa Mohd. Nordin
}

\section{Introduction}

During the 1918 Spanish Flu pandemic, often described as the most devastating epidemic in recorded history, 1 in 5 person was infected and an estimated 50 million lives were lost $^{1}$. The disease was so widespread and pervasive that even the children had a tune which they skipped rope to: I had a little bird, its name was Enza, I opened the window and In-Flu-Enza.

\section{Epidemiology}

Past pandemics and the seasonal influenza have always placed children, especially those less than two years old at increased risk of influenza related morbidity and mortality. Analysis of 7,706 confirmed cases of the Novel Influenza A (H1N1) from 28 countries in the European Union up to 6 June 2009 , showed that $54 \%$ of the cases occurred in children and young adults under 20 years of age $(22 \%$ in children under 10 years $)^{2}$. A report of 642 confirmed cases of Influenza A ( H1N1) in the USA showed that $60 \%$ of cases were in the paediatric population less than 18 years $(20 \%$ in under 10 year olds) $)^{3}$.

This may suggest that the younger population are more biologically susceptible to the virus than older persons. Their mobility and travel may also predispose them to the virus. Some researchers have shown that 1 in 3 persons aged above 60 years have preexisting cross reactive antibodies, which may explain why only $4 \%$ and $5 \%$ in the European and American Influenza A reports respectively, were in those more than 50 years old.

\section{Clinical manifestations}

Children are afflicted by many respiratory illnesses and it may be very difficult to distinguish more common acute respiratory tract infections from the Novel Influenza A infection. Besides, children are less likely to present with the classical symptoms of Influenza A infection, namely high grade fever, sore throat, cough, difficulty breathing, headache and myalgia.

\section{Corresponds to:}

Musa Mohd. Nordin FRCP, FAMM Damansara Specialist Hospital, KualaLumpur, Malaysia. Email: musamn@gmail.com 
Infants may present to the health care worker (HCW) with fever and lethargy, maybe poor feeding and diarrhea and vomiting (acute gastroenteritis being a more common explanation) and no other symptoms or signs related to the respiratory tract. A high index of suspicion is required to make the diagnosis especially if there is a travel history or contact with a case. Interestingly, $25 \%$ in the American and $14 \%$ in the European cohort of Influenza A patients had symptoms (diarrhea and vomiting) related to the gastro-intestinal tract which is not typically found in seasonal influenza.

Unless early diagnosis is made, the child may deteriorate with symptoms and signs of severe disease which includes; cessation of breathing, rapid breathing, difficulty breathing, turning blue, dehydration, altered consciousness and irritability.

\section{Risk factors for severe morbidity or mortality}

A subset of children who are at an even higher risk of influenza related complications includes the following :
a. Less than 2 years old
b. Immunosuppression caused by medications or HIV

c. On long term aspirin therapy

d. Chronic pulmonary, cardiac, hepatic, haematological, neurological, neuromuscular or metabolic disorders

e. Conditions such as cerebral palsy, intellectual and developmental disability, seizure disorders etc which may impair respiratory function.

\section{Transmission and case fatality rates}

The Novel Influenza A (H1N1), which the WHO declared on June 11, 2009, as the causative strain of the first pandemic of the $21^{\text {st }}$ century, bears a disturbing resemblance to the $\mathrm{H} 1 \mathrm{~N} 1$ virus strain which caused the 1918 flu pandemic and is affecting more younger than older people ${ }^{4}$. Transmissibilty of the Novel virus is also much higher than the seasonal influenza, and the estimated rates of human-to-human transmission is similar to the lower estimates of previous influenza pandemics. It has spread within a space of 6 weeks what the previous pandemics could only do in 6 months, facilitated by modern air travel.

Estimates of Case Fatality Rates (CFR) from the Novel Influenza A (H1N1) is lower than the seasonal flu $(0.5-1 \%)$ which is lower than the Spanish Flu (2.5\%) which is lower than the Avian Flu (50\%). The European Centre for Disease Control \& Prevention (ECDC) in its risk assessment report for H1N1 wrote; "a reasonable assumption is a hospitalization rate of $1-2 \%$ and CFR 
of $0.1-0.2 \%^{\prime \prime}$. This was in comparison to earlier estimates of $0.4 \%$ (4 in 1,000) based on data from Mexico ${ }^{6}$. The high estimates from Mexico was probably related to it being a new illness not early and correctly recognized; delay in seeking medical attention and the quality of critical care afforded to the patients who often presented in a moribund condition. In the US alone, there are 200,000 influenza related hospitalizations annually and 36,000 influenza associated deaths during each influenza season. Though the disease severity and mortality rate is much less than the seasonal influenza, the virulence of the Novel Influenza A may change as it mutates and the permissive transmission will further facilitate and present opportunities for the Novel virus to replicate and reassort itself in new host species; potentially evolving into a more lethal virus which would have a significant impact on human history and the global economy.

\section{Containment and mitigation phases}

Global containment of the virus has failed and the WHO has stopped tallying laboratory confirmed cases as the increase is very rapid and the available resources could be better utilized. The most effective strategy to break this exponential chain of transmission and control the epidemic is through a mass vaccination program. The first pandemic vaccine trial in humans are underway in Australia and if found to be safe and effective, a mass immunization program will be launched, earliest before the end of $2009^{7}$.

\section{Special considerations for children}

Meanwhile, during the mitigation phase, various universal influenza pandemic preparedness programs have been implemented to decrease the global impact of this Novel virus. Measures specific to the health and lives of children include :

a. Focusing on children's hygiene is one of the best way to reduce transmission of the flu virus. This is probably because children are very sociable and enjoys the most amount of physical contact with other people. Encouraging parents, teachers and day care workers to promote frequent hand washing and other good hygiene practices would go a long way towards mitigating this outbreak. In Pittsburgh, during the Spanish Flu pandemic, school children were given information to take home and warned not to gather in groups . A review of several published studies showed that frequent hand washing, using gloves, gowns and masks with filtration, and isolating probable cases 
helped to reduce transmission of viral respiratory diseases.

b. Children with confirmed or probable Influenza A infection and not sick enough to warrant hospital care should be home quarantined and all related public health measures should be strictly adhered.

c. Hospitals and Health Care Workers (HCW) are bearing the brunt of this added clinical burden. Triage at the entry points of all healthcare facilities are meant to timely identify suspected cases for clinical management and decrease the risk of transmission to other patients and $\mathrm{HCW}$. Children should not be brought to hospitals and other healthcare facilities unless they are sick and require specific treatment. Do not be intimidated by $\mathrm{HCW}$ who are adorning masks, gowns and gloves because they are working in a high risk environment and require them for their personal protection. During the onset of the first outbreak in Mexico, in March-April 2009, 22 HCW developed influenza like illness (ILI) within 7 days of contact with the index patient and required treatment with oseltamivir ${ }^{8}$. d. A whole host of nonpharmacological interventions were undertaken in 1918 to mitigate the impact of the influenza pandemic ${ }^{9}$. These included the wearing of surgical masks, encouraging people to stay home, prohibition of public gatherings and the closure of schools. St. Louis in the US, which implemented an early and sustained strategy of school closures and cancellation of public gatherings did not experience as severe an outbreak when compared to other US cities. The WHO has recommended that the closure of schools is one of the mitigation measures that should be considered by countries if the pandemic continues to worsen.

e. Children are more likely to be sicker and die from the seasonal influenza than the Novel Influenza A. The seasonal influenza is preventable and all children should continue to get their annual seasonal flu shots. It is not expected to provide any substantial protection against the Novel Influenza A virus. The US CDC recently extended the use of the influenza vaccine to children up to 18 years from the previous six 
months to five year olds. Those above 65 years and persons with co-morbidities were similarly listed as high priority for the influenza vaccination.

f. Parents should make sure that their children's other immunizations are up to date. Many of the deaths during the 1918 pandemic were not directly caused by the H1N1 virus but were due to secondary bacterial pneumonia which set in after the virus had weakened the body's defences ${ }^{10}$. The most common bacteria isolated from antemortem and post-mortem specimens were the pneumococcus $\quad(50 \%)$, haemophilus influenza (25\%), staphylococcus aureus and meningococcus. The pneumococcal conjugate vaccine (PCV), the 23-valent pneumococcal vaccine, the Hib vaccine and the quadrivalent meningococcal vaccine would help prevent against a big proportion of the killer copathogens ${ }^{11}$.

g. Because children are particularly susceptible to the new flu virus, HCW may need to be especially alert to secondary bacterial infections in their narrower airways. The advent and availability of effective and affordable antibiotics against these bacterial superinfections has helped to reduce complications from influenza. If a child has had the flu for five to seven days and is not improving or getting worse, this is one situation where doctors would need to seriously consider prescribing appropriate antibiotics.

h. The Novel Influenza A (H1N1) virus is susceptible to the anti-virals, oral oseltamivir and inhaled zanamivir. It is most effective when commenced within 48 hours of developing ILI and administered for five days $^{12}$. Notwithstanding, many patients with Influenza A (H1N1) have recovered spontaneously without anti-viral treatment. The most common adverse effects of oseltamivir are nausea and vomiting which improves by taking it with food. Children with severe clinical illness, radiological abnormalities and other comorbidities as outlined earlier should be considered for early anti-viral therapy.

i. Sometimes antivirals are given to otherwise well children 
who are known or strongly suspected to have been exposed to another person with the Novel Influenza A virus. This chemoprophylaxis with antivirals is to prevent the child from becoming infected with the virus or to make the infection milder. Prophylactic oseltamivir is approved for children 12 months or older and should be started upon exposure and continued for 7 to 10 days, at a lower dose than for therapy. Zanamivir is approved for chemoprophylaxis in children 5 years or older.

\section{Conclusion}

Since its first appearance in April 2009, at the US-Mexican border, the Novel Influenza A (H1N!) has spread to over
160 countries, infected well in excess of 200,000 people and claimed more than 800 lives $^{12}$. The WHO on June 11, 2009, declared an influenza pandemic caused by this Novel strain. Children and young adults are more susceptible to the Novel strain than the elderly population. Though the clinical syndrome is relatively less severe than the seasonal flu, the human-human transmission of the strain is universal and exponential. Global Influenza Pandemic Preparedness plans are in place to mitigate the human and socioeconomic impact of this Novel flu virus. No vaccine is presently available to prevent infection with the Novel virus, break its chain of transmission and to contain and control the epidemic. Human trials with the pandemic vaccine are now in progress and should be available for large scale immunization before the close of 2009

\section{References}

1. Johnson NPAS, Mueller J. Updating the accounts: global mortality of the 1918-1919 Spanish Influenza pandemic. Bull Hist Med 2002; 76:105-115.

2. ECDC Surveillance Report. Analysis of influenza A (H1N1) individual case reports in EU and EEA countries. 10 July 2009.
3. Novel Swine-Origin Influenza A (H1N1) Virus Investigation team. Emergence of a novel swine origin influenza A (H1N1) virus in humans. $N$ Eng $J$ Med 2009; 360:2605-15.

4. Taubenberger JK, Morens DM. 1918 Influenza: the mother of all pandemics. Emerg

Infect Dis 2006; 12:15-22. 
5. ECDC Interim Risk Assesment. Influenza A (H1N1) 2009 Pandemic. 20 July 2009.

6. Fraser C. et al. Pandemic potential of a strain of influenza A (H1N1): early findings. Science 2009 May 14.

7. 7.Isabel Leroux-Roels, Geert Leroux-Roels. Current status and progress of prepandemic and pandemic influenza vaccine development. Expert Review of Vaccines, April 2009. 8 (4): 401423

8. Rogelio Perez-Padilla et al. Pneumonia and respiratory failure from Swine-Origin Influenza A (H1N1) in Mexico. $N$ Eng $J$ Med 2009; 361:680-689

9. Markel $\mathrm{H}$ et al. Non-pharmaceutical interventions implemented by US cities during the 1918-1919 influenza pandemic. JAMA 2007; 298(6): 644-654
10. Harsch EF, McKinney M. Epidemic Bronchopneumonia at CampGrant, ILL. Preliminary Report. JAMA 1918; 71:1735-36

11. Hall JN, Stone MC, Simpson JC. The Epidemic of Pneumonia Following Influenza at Camp Logan, Texas: Preliminary Report. JAMA 1918; 71:1986-87

12. ECDC Interim Guidance. Public health use of influenza anti-virals during influenza pandemics. June 2009 